\title{
Construction of an algorithm for the analytical solution of the Kolmogorov-Feller equation with a nonlinear drift coefficient
}

\author{
Andrei Firsov ${ }^{1}$, Anton Zhilenkov ${ }^{2}$ \\ ${ }^{1}$ Peter the Great St. Petersburg Polytechnic University, Saint Petersburg, Russia \\ ${ }^{2}$ Saint Petersburg State Marine Technical University, Saint Petersburg, Russia \\ ${ }^{2}$ Corresponding author \\ E-mail: ${ }^{1}$ anfirs@yandex.ru, ${ }^{2}$ marine_electronics@corp.smtu.ru
}

Received 12 May 2019; accepted 18 June 2019

DOI https://doi.org/10.21595/vp.2019.20799

Check for updates

Copyright $(2019$ Andrei Firsov, et al. This is an open access article distributed under the Creative Commons Attribution License, which permits unrestricted use, distribution, and reproduction in any medium, provided the original work is properly cited.

\begin{abstract}
The paper proposes a constructive method for solving the stationary Kolmogorov-Feller equation with a nonlinear drift coefficient. The corresponding algorithms are constructed and their convergence is justified. The basis of the proposed method is the application of the Fourier transform.
\end{abstract}

Keywords: mathematical model, analytical solution, Kolmogorov-Feller equation, nonlinear drift coefficient, constructive method for solving.

\section{Introduction}

This paper proposes an approach to constructing solutions of differential equations of fractional order of the Kolmogorov-Feller type. We consider equations with nonlinear coefficients, namely the case of a quadratic dependence of the drift coefficient on the independent variable. As far as we know, this method of construction is not presented in the literature. The advantage of the method is its effectiveness in numerical implementation.

\section{Mathematical model of the problem}

Let's consider the form of the Kolmogorov-Feller Eq. (1) with the drift coefficient $\beta \neq 0$, which depends nonlinearly on the coordinate:

$\frac{d}{d x}\left[\left(\alpha x+\beta x^{2}\right) W(x)\right]+v \int_{-\infty}^{+\infty} p(A) W(x-A) d A-v W(x)=0, \quad-\infty<x<+\infty$.

In the literature, it is customary to consider the simplified case $\beta=0$. In our case for normal form we have:

$W(x) \underset{\substack{x \rightarrow \pm \infty \\ x \rightarrow \infty}}{\longrightarrow} \quad \int_{-\infty}^{+\infty} W(x) d x=1$,
$p(A) \underset{|A| \rightarrow \infty}{\longrightarrow} 0, \quad \int_{-\infty}^{+\infty} p(A) d A=1$.

We assume $p(A)$ - analytical function and $\hat{p}(k)=\int_{-\infty}^{+\infty} p(x) e^{i x k} d x-$ it's Fourier transform, where $|A|<R$ or:

$\hat{p}(k)=\hat{p}_{0}+\hat{p}_{1} k+\hat{p}_{2} k+\ldots, \quad|k|<k_{0}, \quad k_{0} \gg 1$.

Insofar as Eqs. (2-3), we have: 
$\hat{p}_{0}=\hat{p}(0)=1$.

In case $p(x)$ - even function, we have $\hat{p}_{2 s-1}=0, s=1,2, \ldots$, and $\hat{p}(k)$ - is real analytical function. From Eq. (2) we have:

$\left\{\begin{array}{l}\int_{-\infty}^{+\infty}|\widehat{W}(k)| d k<\infty, \\ \widehat{W}(0)=1 .\end{array}\right.$

Obviously, we can go from solving the Eq. (1) with Eq. (2), to the equation:

$i \beta \widehat{W^{\prime \prime}}(k)-\alpha \widehat{W^{\prime}}(k)+v \rho(k) \widehat{W}(k)=0$.

From Eq. (6) we have:

$\rho(0)=\hat{p}_{1}=\int_{-\infty}^{+\infty} x p(x) d x$,

$\rho(k)=\hat{p}_{1}+\hat{p}_{2} k+\hat{p}_{3} k^{2}+\ldots, \quad|k|<k_{0}$.

Again, since $\hat{p}(k) \rightarrow 0,|k| \rightarrow \infty$, we get:

$\rho(k) \sim \frac{-1}{k}, \quad(|k| \rightarrow \infty)$.

\section{Mathematical model analysis}

For:

$\widehat{W}(k)=\varphi(k) e^{-\int_{0}^{k} \psi(k) d k}$,

we get:

$\varphi^{\prime \prime}+\left(-2 \psi+i \frac{\alpha}{\beta}\right) \varphi^{\prime}+\left(\psi^{2}-\psi^{\prime}-i \frac{\alpha}{\beta} \psi-i \frac{v}{\beta} \rho\right) \varphi=0$.

Putting:

$\psi=i \frac{\alpha}{2 \beta}$,

we'll get for $\varphi(k)$ following equation:

$\varphi^{\prime \prime}-q(k) \varphi=0$,

where:

$\varphi(k)=\widehat{W}(k) e^{i \frac{\alpha}{2 \beta} k}$,

$q(k)=-\frac{\alpha^{2}}{2 \beta^{2}}+i \frac{v}{\beta} \rho(k)$.

For $q(k)$ we can highlight some properties.

1) From Eq. (10) it follows: 
$q(k) \rightarrow-\frac{\alpha^{2}}{2 \beta^{2}}, \quad|k| \rightarrow \infty$

2) From Eq. (19) we have:

$q(k)=-\frac{\alpha^{2}}{2 \beta^{2}}+i \frac{v}{\beta} \hat{p}_{1}+i \frac{v}{\beta}\left(\hat{p}_{2} k+\hat{p}_{3} k^{2}+\ldots\right), \quad|k|<k_{0}$,

or

$q(k)=q_{0}+q_{1} k+q_{2} k^{2}+\ldots, \quad|k|<k_{0}$,

where:

$\left\{\begin{array}{l}q_{0}=-\frac{\alpha^{2}}{2 \beta^{2}}+i \frac{v}{\beta} \hat{p}_{1}, \\ q_{n}=i \frac{v}{\beta} \hat{p}_{n+1} .\end{array}\right.$

3) Also:

$q(k)=-\delta(k)+i \frac{v}{\beta} \operatorname{Re} \rho(k)$.

Lemma 1. For $\sqrt{q(k)}$ :

$$
\begin{gathered}
(\sqrt{q(k)})_{1}=\sqrt{|q(k)|}\left\{\frac{1}{2}\left(1+\left(1+\frac{[\operatorname{Re} \rho(k)]^{2} v^{2}}{\delta^{2} \beta^{2}}\right)^{-1 / 2}\right)\right\}^{1 / 2} \\
-i \sqrt{|q(k)|}\left\{\frac{1}{2}\left(1-\left(1+\frac{[\operatorname{Re} \rho(k)]^{2} v^{2}}{\delta^{2} \beta^{2}}\right)^{-\frac{1}{2}}\right)\right\}^{1 / 2},
\end{gathered}
$$

is $C^{2}$ by $k \in(0,+\infty)$ and $\left.\operatorname{Re}(\sqrt{q(k)})\right)_{1}>0$ for $k$, which are large enough.

\section{Construction of the solution of the transfer theory problem}

We will use the well-known asymptotic theorem for solving the equation:

$u^{\prime \prime}(x)-q(x) u(x)=0$,

when $x \rightarrow+\infty$.

Theorem 1. Let in the Eq. (21) $q(x) \in C^{2}(0, \infty), q(x) \neq 0$ for sufficiently large $x$ and let there exist a branch $\sqrt{q(x)}$ of class $C^{2}(b, \infty)$ such that $\operatorname{Re} \sqrt{q(x)}>0, x>b \geq 0$. Let further $\alpha_{1}(x)=\frac{1}{8} \frac{q^{\prime \prime}}{q^{3 / 2}}-\frac{s}{32} \frac{\left[q^{\prime}\right]^{2}}{q^{5 / 2}}$ and $\int^{\infty}\left|\alpha_{1}(x)\right| d x<\infty$. Then Eq. (21) has a solution:

$u(x)=q^{-\frac{1}{4}}(x) e^{-\int^{x} \sqrt{q(t)} d t}\left[1+\varepsilon_{2}(x)\right], \quad \varepsilon_{2}(x) \rightarrow 0, \quad(x \rightarrow \infty)$.

Moreover, for $x>0$ : 
$\left|\frac{u(x)}{\tilde{u}(x)}-1\right| \leq 2\left(e^{2 \int_{x}^{\infty}\left|\alpha_{1}(t)\right| d t}-1\right)$

$\left|\frac{u^{\prime}(x)}{\sqrt{q(x)} \tilde{u}(x)}+1\right| \leq \frac{1}{4}\left|\frac{q^{\prime}(x)}{q^{\frac{3}{2}}(x)}\right|+4\left(1+\frac{1}{4}\left|\frac{q^{\prime}(x)}{q^{\frac{3}{2}}(x)}\right|\right) \times\left(e^{2 \int_{x}^{\infty}\left|\alpha_{1}(t)\right| d t}-1\right)$.

If $\frac{q^{\prime}(x)}{q^{\frac{3}{2}}(x)} \rightarrow 0,(x \rightarrow \infty)$, then $u^{\prime}(x)=q^{1 / 4}(x) e^{-\int^{x} \sqrt{q(t)} d t}\left(1+\varepsilon_{1}(x)\right), \varepsilon_{1}(x) \rightarrow 0, x \rightarrow+\infty$.

Lemma 2. If $\left|\widehat{p}^{\prime}(k)\right| \leq O\left(\frac{1}{k}\right)$ and $\left|\widehat{p^{\prime \prime}}(k)\right| \leq O\left(\frac{1}{k}\right)$, then for Eq. (12) the previous theorem is valid.

Thus, further we solve the following problem:

$\varphi^{\prime \prime(k)}-q(k) \varphi(k)=0, \quad k>0$,

$\int \varphi(0)=1$,

$\left\{\begin{array}{l}\varphi(k) \rightarrow 0, \quad k \rightarrow+\infty\end{array}\right.$

Here $q(k)$ is given by Eq. (15). Further, we assume that the assumptions of Theorem 1 are fulfilled. In particular, the function $q(k)$ is analytic when $|k|<k_{0}, k_{0} \gg 1$ (see Eq. (18a)).

From the theory of differential equations, we obtain for the coefficients $a_{n}$ following infinite system of equations:

$\left\{\begin{array}{l}(n+1)(n+2) a_{n+2}-\sum_{s=0}^{n} a_{s} q_{n-s}=0, \quad n=0,1,2, \ldots \\ a_{0}=1\end{array}\right.$

For $a_{2}$ we immediately get at $n=0$ :

$a_{2}=\frac{1}{2} q_{0}=-\frac{\alpha^{2}}{4 \beta^{2}}+i \frac{v}{2 \beta} \hat{p}_{1}$

In case of even $p(x): \hat{p}_{1}=0, a_{2}=-\frac{\alpha^{2}}{4 \beta^{2}}$. The determinant of the matrix $A_{N}$ of this system is:

$$
\begin{aligned}
\Delta_{N} & =\operatorname{det} A_{N}=(2 \cdot 3)(3 \cdot 4) \ldots(N+1)(N+2) \\
& =\frac{1}{2}(N+1) !(N+2) !=\frac{N+2}{2}[(N+1) !]^{2}>0 .
\end{aligned}
$$

In these designations for $\varphi(k)$ we have the expression:

$$
\begin{aligned}
& \varphi(k)=1+a_{1} k+a_{2} k^{2}+h(k)+a_{1} g(k)=a_{1}(k+g(k))+1+a_{2} k^{2}+h(k) \\
& \quad \equiv a_{1} g_{1}(k)+h_{1}(k),
\end{aligned}
$$

where $k+g(k)=g_{1}(k), 1+a_{2} k^{2}+h(k)=h_{1}(k)$.

To find the coefficient $a_{1}$, we use the asymptotic solution $\varphi(k)(k \rightarrow+\infty)$, given by Theorem 1. Let $k_{1}<k_{0}$. Then by Theorem 1 we get:

$$
\left\{\begin{array}{l}
a_{1} g_{1}\left(k_{1}\right)+h_{1}\left(k_{1}\right)=C q^{-1 / 4}\left(k_{1}\right)\left(1+\varepsilon_{2}\left(k_{1}\right)\right), \\
a_{1} g_{1}^{\prime}\left(k_{1}\right)+h_{1}^{\prime}\left(k_{1}\right)=-C q^{1 / 4}\left(k_{1}\right)\left(1+\varepsilon_{1}\left(k_{1}\right)\right) .
\end{array}\right.
$$

If $k_{1} \gg 1$, then $\left|\varepsilon_{1}\left(k_{1}\right)\right| \ll 1,\left|\varepsilon_{2}\left(k_{1}\right)\right| \ll 1[7,8]$. Therefore, Eq. (28) can be approximately replaced by the system: 
$\left\{\begin{array}{l}\tilde{a}_{1} g_{1}\left(k_{1}\right)+h_{1}\left(k_{1}\right)=\tilde{C} q^{-1 / 4}\left(k_{1}\right) \\ \tilde{a}_{1} g^{\prime}{ }_{1}\left(k_{1}\right)+h^{\prime}{ }_{1}\left(k_{1}\right)=-\tilde{C} q^{1 / 4}\left(k_{1}\right)\end{array}\right.$

where $\tilde{a}_{1}$ and $\tilde{C}$ are approximate values for $a_{1}$ and $C$. From Eq. (29) we find:

$$
\left\{\begin{array}{l}
\tilde{a}_{1}=-\frac{h_{1} q^{1 / 2}+h^{\prime}{ }_{1}}{g_{1} q^{1 / 2}+g^{\prime}{ }_{1}} \\
\tilde{C}=q^{1 / 4} \frac{g^{\prime}{ }_{1} h_{1}-g_{1} h^{\prime}}{g_{1} q^{1 / 2}+g^{\prime}}{ }_{1}
\end{array}\right.
$$

where all functions are calculated when $k=k_{1}$. For an approximate value $\tilde{\varphi}(k)$ of $\varphi(k)$ we therefore have:

$$
\left\{\begin{array}{l}
\tilde{\varphi}(k)=\left\{\begin{array}{l}
\tilde{a}_{1} g_{1}(k)+h_{1}(k), \quad 0 \leq k \leq k_{1}, \\
\left.\left(\frac{g^{\prime} h_{1}-g_{1} h_{1}^{\prime}}{g_{1} q^{\frac{1}{2}}+g^{\prime}{ }_{1}}\right)\right|_{k=k_{1}} q^{\frac{1}{4}}\left(k_{1}\right) q^{-\frac{1}{4}}(k) e^{-\int_{k_{1}}^{k} \sqrt{q(t)} d t}, \quad k \geq k_{1}, \\
k_{1} \gg 1, \quad k_{1}<k_{0},
\end{array}\right. \\
\tilde{\varphi}(-k)=\frac{\tilde{\varphi}(k), \quad k \geq 0 .}{\tilde{\tilde{\varphi}}(k)}
\end{array}\right.
$$

\section{Results and conclusions}

For construction of the analytical solution of the Kolmogorov-Feller Eq. (1) one can use the following algorithm.

1) Take the desired function $\varphi(k)=\widehat{W}(k) e^{i \frac{\alpha}{2 \beta} k}$.

2) For $\varphi(k)$ we have $\varphi^{\prime \prime}(k)-q(k) \varphi(k)=0, k>0$ under:

$\left\{\begin{array}{l}\varphi(0)=1, \\ \varphi(k) \rightarrow 0, \quad k \rightarrow+\infty,\end{array}\right.$

$q(k)=q_{0}+q_{1} k+q_{2} k^{2}+\ldots, \quad|k|<k_{0}$.

3) We can get $q_{j}$ from:

$$
\left\{\begin{array}{l}
q_{0}=-\frac{\alpha^{2}}{2 \beta^{2}}+i \frac{v}{\beta} \hat{p}_{1}, \\
q_{n}=i \frac{v}{\beta} \hat{p}_{n+1},
\end{array}\right.
$$

where $\hat{p}_{j}-$ are from $\hat{p}_{s}=\frac{\hat{p}^{(s)}(0)}{s !}=\frac{1}{s !}(i)^{s} \int_{-\infty}^{+\infty} x^{s} p(x) d x$, or from $\hat{p}(k)=\int_{-\infty}^{+\infty} p(x) e^{i x k} d x$ with $\hat{p}(k)=\hat{p}_{0}+\hat{p}_{1} k+\hat{p}_{2} k+\ldots,|k|<k_{0}, k_{0} \gg 1$.

4) Then we have solution in form $\varphi(k)=1+a_{1} k+a_{2} k^{2}+\ldots, 0 \leq k<k_{0}$, where $a_{j}, j \geq 2$ are determined from equations:

$(n+1)(n+2) a_{n+2}-\sum_{s=0}^{n} a_{s} q_{n-s}=0, n=0,1,2, \ldots, \quad a_{0}=1$,

and: 
$a_{1}=-\lim _{k \rightarrow+\infty} \frac{h_{1}(k) q^{\frac{1}{2}}(k)+{h^{\prime}}_{1}(k)}{g_{1}(k) q^{\frac{1}{2}}(k)+g^{\prime}{ }_{1}(k)}$

where $h_{1}(k), g_{1}(k)$ are determined from Eqs. (30), (31).

\section{References}

[1] Kim Ju Gyong, Choe Il Su A solution to Kolmogorov-Feller equation and pricing of option. International Symposium in Commemoration of the 65th Anniversary of the Foundation of Kim Il Sung University (Mathematics), 2011.

[2] Blackledge J., Lamphiere M., Panahi A. Simulation and analysis of stochastic signals using the Kolmogorov-Feller equation. IET Irish Signals and Systems Conference, 2012.

[3] Popov A. V., Seredenko N. A., Zhilenkov A. A. Control system of multi-level manipulator with rotational degrees of mobility. Proceedings of the IEEE Conference of Russian Young Researchers in Electrical and Electronic Engineering, 2019.

[4] Zhilenkov A. High productivity numerical computations for gas dynamics modelling based on DFT and approximation. IEEE Conference of Russian Young Researchers in Electrical and Electronic Engineering, 2019.

[5] Nyrkov A. P., Chernyi S. G., Sokolov S. S., Zhilenkov A. Optimization problem of thermal field on surface of revolving susceptor in vapor-phase epitaxy reactor. IOP Conference Series: Earth and Environmental Science, Vol. 87, Issue 8, 2017, p. 082060. 